\title{
Why aggregate? On the evolution of aggregative multicellularity
}

\author{
Marco La Fortezza, Kaitlin A. Schaal and Gregory J. Velicer \\ Institute for Integrative Biology, ETH Zürich, 8092 Zürich, Switzerland \\ Corresponding Author: marco.lafortezza@env.ethz.ch
}

\begin{abstract}
Microbes have evolved many fascinating and complex ways of interacting with conspecifics. Perhaps one of the most interesting is aggregative multicellularity, wherein independent cells come together and adhere to one another in order to form a larger entity. The fundamental benefits of active aggregation into multicellular groups generally remain unclear, and there are many open questions about what selective pressures led to the evolution of this behavior in various eukaryotic and prokaryotic taxa, most notably the dictyostelids and the myxobacteria. Aggregative multicellularity can be partitioned into three main phases: coming together, staying together as a group, and disaggregation. Different selective pressures may have led to adaptations unique to each phase. While aggregative microbial systems generally form elevated multicellular structures such as fruiting bodies, these can vary in complexity and morphology even among closely related species. What evolutionary forces shaped such morphological diversification remains unknown. Strains that are not genetically identical can coaggregate, which can impact group-level function either positively through functional synergy or negatively through harmful exploitation. Such chimerism within aggregates is likely to have played important roles in shaping the evolution of microbial multicellularity. Much further research is needed into the evolutionary forces and processes leading to and shaping the many forms of microbial aggregation.
\end{abstract}

Keywords: eco-evo-devo, evolution of multicellularity, fruiting body, life-history evolution, microbial evo-devo

\section{Introduction}

Microbes have evolved many fascinating and complex behaviors. They were long thought of as self-sufficient single cells that compete with each other for resources, but in fact many of their interactions benefit each other. In particular, some microbes can actively aggregate into cooperative multicellular groups. This ability has evolved independently multiple times throughout the tree of life, suggesting that it provides particular benefits to the organisms which possess it. Obligately multicellular organisms have also evolved aggregative behaviors (see Box 1), but here we focus on aggregation among reproductively autonomous cells.

Microbes can form a variety of multicellular associations, including filaments, biofilms, and fruiting bodies (Grosberg and Strathmann 2007; Claessen et al. 2014). Filaments form when newly divided cells adhere to one another rather than separating (Flores and Herrero 2010; Flärdh et al. 2012). Biofilms form when cells stick to each other and then attach to a surface. This can involve clonal adhesion, as in filament formation, but the biofilm may also passively recruit external cells (passive aggregation) (Smith et al. 2015; Trunk et al. 2018), or these cells may actively join the biofilm using directed motility (active aggregation) (Houry et al. 2010). We do not cover microbial filaments or biofilms in this chapter because active aggregation is not the primary force driving the formation of either. Fruiting bodies, however, are raised or extended cell mounds generated primarily by active aggregation and within which cells often differentiate into stress-resistant spores.

Fruiting body formation has been documented in four Eukarya supergroups: Amoebozoa (Dictyostelia and Copromyxa), Discoba (Acrasidae), Holozoa (Fonticula), and SAR/Harosa (Sorodiplophrys in Stramenopiles, Sorogena in Alveolata, and Guttulinopsis in Rhizaria) (Figure 1) (Sugimoto \& Endoh 2006; Brown, Spiegel, and Silberman 2012; Brown et al. 2012b; Brown and Silberman 2013). These organisms can be found in a variety of environments (Swanson, Vadell and Cavander 1999) and have similar multicellular life cycles. Their process of aggregation often results in fruiting bodies (sometimes known as sorocarps), whose morphologies can differ greatly across species 
(see Chapter 8 and Figure 1). Of these taxa, Dictyostelia is the best described, thanks to the well-known model species Dictyostelium discoideum (Kessin 2001; Bonner 2009).

Some Bacillus species (Branda et al. 2001; Claessen et al. 2014) form fruiting bodies, but the most famous bacterial fruiting bodies are those formed by species in the order Myxococcales. The myxobacteria, or "slime bacteria," were first identified by Roland Thaxter (Thaxter 1892), who found and examined fruiting bodies on decaying wood. The best known myxobacterium is Myxococcus xanthus (Yang and Higgs 2014). In addition to being models for studies of aggregative development, both $D$. discoideum and $M$. xanthus have become model experimental systems for studies of the ecology and evolution of microbial social behaviors (Velicer and Vos 2009; Medina et al. 2019).
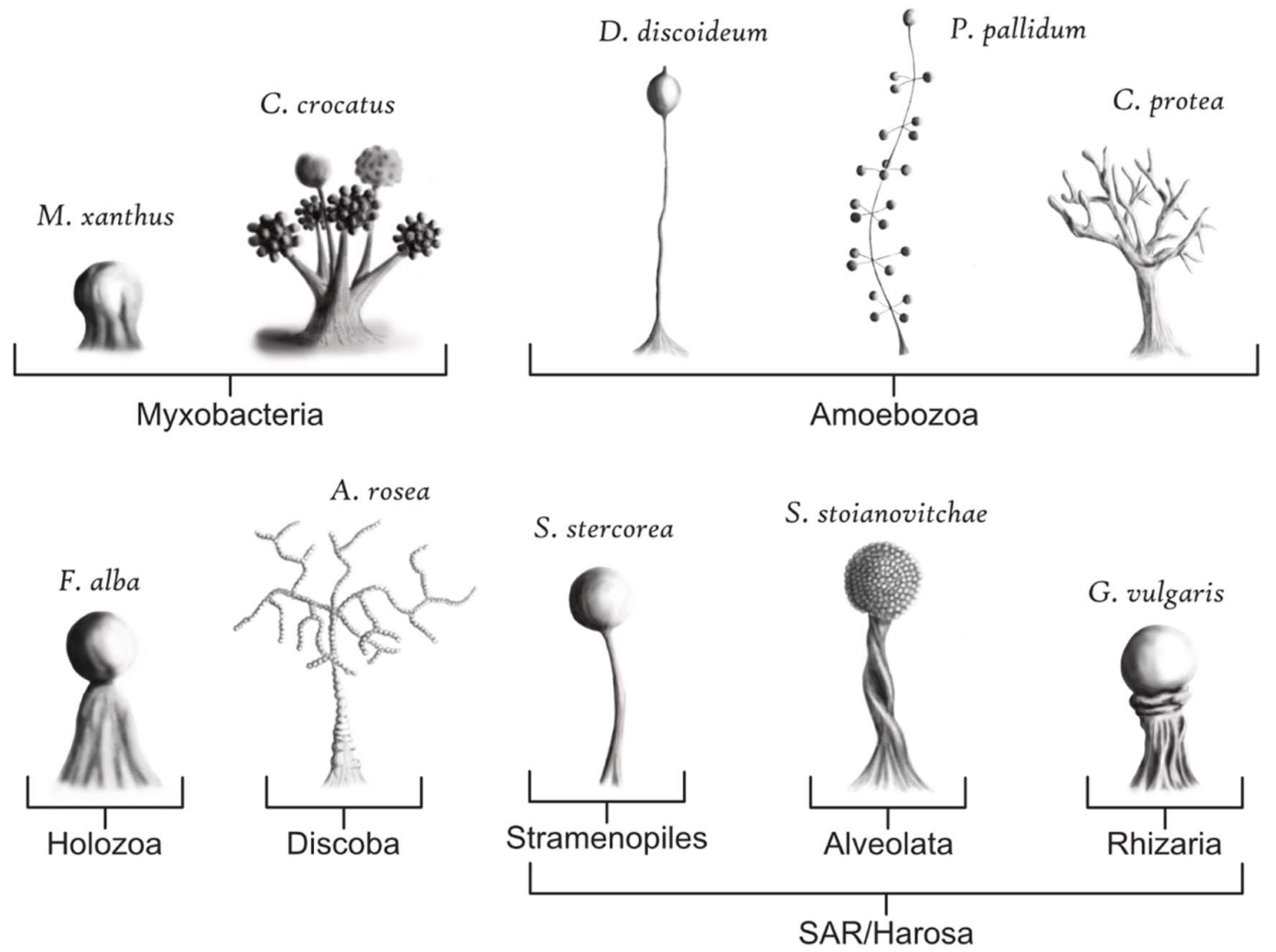

Figure 1. Representative examples of fruiting body morphologies across different taxa. Drawings are at different scales and are based on: $M$. xanthus and C. crocatus (direct observations), D. discoideum (direct observation), $P$. pallidum (Kawabe et al. 2015), C. protea (Brown and Silberman 2013), F. alba (Brown and Silberman 2013 ), A. rosea (Brown, Spiegel, and Silberman 2012), G. vulgaris (Brown and Silberman 2013), S. stoianovitchae (Olive and Blaton 1980), S. stercorea (Dykstra and Olive, 1975).

Active aggregation seems to be adaptive. Aggregative behaviors are energetically costly and are regulated by complex genetic pathways, so they presumably evolved via selection. However, other evolutionary processes may have played a role. In this chapter, we consider why aggregative multicellularity might be adaptive and which features may be nonadaptive. We discuss evolutionary hypotheses for the origin, maintenance, and complexification of several diverse forms of aggregative multicellularity, focusing on systems that use motility to aggregate. The chapter is organized according to three temporal phases of aggregate life cycles: first, the process of active aggregation, or coming together; second, life in the aggregate; and third, disaggregation and post-aggregate behavior (Figure 2 ). It ends with a discussion of chimeric aggregates (those composed of multiple genotypes) and an outlook on the broader relevance of studying this intriguing phenomenon.

\section{Coming together}

Multicellular aggregates begin when cells seek each other out and stick together, increasing their local density. We consider two types of forces at work during this process: those responsible for 
increasing cell density and those responsible for maintaining it. Cell density can be increased by physical forces external to the aggregating cells, in which case this step of aggregation is passive (Angel et al. 2020). For example, cells can encounter and stick to one another as they are transported by flowing liquid. Alternately, cells can aggregate by motility, an agent-like behavior (Angel et al. 2020). In this case, cells call and search for each other by producing and responding to chemical cues or signals ( Du et al. 2015; Kroos 2017). We will focus on forms of aggregative multicellularity in which cells use motility to actively increase their local density, such as fruiting body formation (Claessen et al. 2014; Du et al. 2015), rather than those generated more passively, such as flocculation of planktonic yeast cells (Verstrepen and Klis 2006; Trunk 2018). Regardless of whether cell density increases passively or actively, cells will maintain high density under physical disturbance only if they establish stable physical contacts by producing cell-cell adhesins or by using chemoattraction and motility behavior to remain near one another, both of which constitute active behavior.

Aggregation in myxobacteria and dictyostelids. The myxobacteria and the dictyostelids are by far the most studied taxa in which motility-driven aggregation has evolved. In both, cells in sufficiently

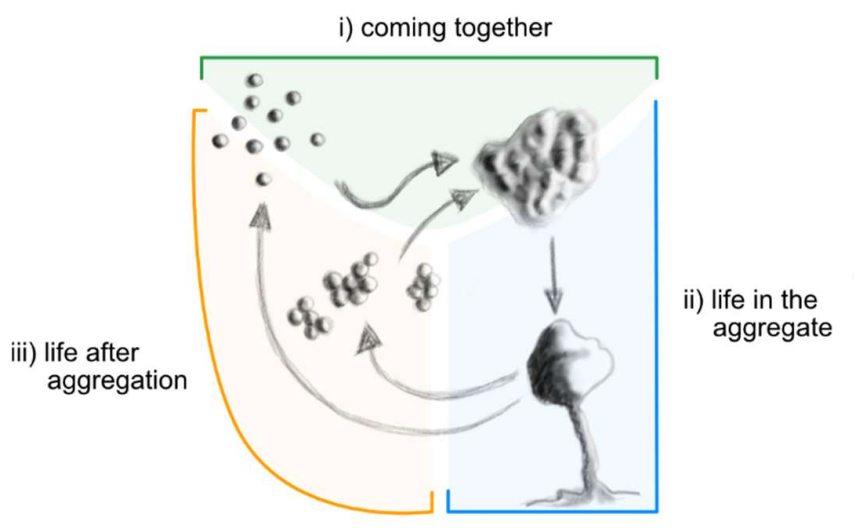

Figure 2. Stereotypical life cycle of aggregative multicellular organisms divided into three major stages. i) coming together: Single cells and/or cell groups actively aggregate and adhere to one another to form a multicellular structure. ii) life in the aggregate: Cells interact within the aggregate while group-level behaviors and morphologies become more pronounced. In the drawing, an early multicellular aggregate develops into a mature fruiting body. In some cases, the aggregate may be dispersed as a whole to a new location by an external force. In other cases, the aggregate may not be dispersed before environmental conditions which previously favored aggregation change to again favor growth and disaggregation. iii) life after aggregation: Once spores have been exposed to nutrients (e.g., prey) and germinated, cells may reduce local cell density by migrating away from the aggregate before living asocially or (e.g., dictyostelids) or in cooperative groups (e.g., myxobacteria) until the next cycle of aggregation.

dense populations respond to nutrient deprivation by aggregating into cell mounds. These increase in size and, at least in some species, in morphological complexity as more cells join and development proceeds, culminating in the formation of a fruiting body. Some cells die during development, while others differentiate into stress-resistant spores (Stenhouse and Williams 1977; Wireman and Dworkin 1977; O'Connor and Zusman 1988; Kessin 2001; Lee et al. 2012). Other fruiting body forming eukaryotes aggregate in a similar fashion, with a few exceptions. For example, the Copromyxa protea fruiting body is composed entirely of living cells, which one by one move to the top of the structure and encyst (Spiegel 1978).

In the myxobacteria, mounds of aggregated cells gradually morph into elevated fruiting bodies with species-specific phenotypes (Grilione and Pangborn 1975; Spröer, Reichenbach, and Stachenbrandt 1999). Within fruiting bodies of M. xanthus, cells may die, convert to spherical spores, or remain rodshaped. Development in $M$. xanthus involves complex regulatory pathways and multiple temporally regulated inter-cellular signals (Kroos 2017). The highly conserved bacterial stringent response, in which nutrient deprivation induces (p)ppGpp synthesis (Kroos 2017), has been evolutionarily integrated with a myxobacteria-specific quorum-sensing system, and the combination triggers aggregative motility 
behavior upon depletion of extracellular amino acids. In M. xanthus, the quorum signal that triggers aggregation is a mix of amino acids derived from degrading cell-surface peptides; this has been termed the A signal (Kaplan and Plamann 1996). Simple aggregation of cells in response to A signal may have been an early adaptation in the evolution of myxobacterial multicellularity prior to the evolution of more complex structures, though so far there are no studies which examine this hypothesis.

Aggregating and sporulating usually happen together. However, some M. xanthus genotypes sporulate extensively without aggregating (Velicer, Kroos and Lenski 1998; La Fortezza and Velicer 2021), which raises the question of whether sporulation evolved prior to aggregation. Which of the genes necessary for spore production are also necessary for fruiting body formation remains to be fully determined. Many myxobacterial species actively aggregate prior to sporulating rather than simply sporulating in place in response to starvation cues, which could be done immediately and without expending energy on aggregating, suggesting that there are adaptive benefits to sporulating within a fruiting body. Additionally, most $M$. xanthus mutants that are defective at aggregation are also to some degree defective at spore production, indicating deep evolutionary integration of these processes.

In the dictyostelids, cells in nutrient-rich environments appear to interact with each other rarely and briefly (Kessin 2001). They usually feed on bacteria, which they find by following traces of folic acid that prey cells release into their surroundings (Pan, Hall, and Bonner 1972). However, when prey are scarce, dictyostelid cells become social. This transition to multicellularity can be easily induced and observed in the lab by manipulating nutrient concentrations. $D$. discoideum cells secrete cAMP to actively attract each other, though the role of cAMP is not limited to chemotactic aggregation (Du et al. 2015, Meena and Kimel 2017, Kawabe et al. 2019) and different species of amoebae use cAMP at different stages of aggregation (Kawabe et al. 2019). As for the myxobacteria, there seems to be a dearth of dictyostelid studies testing for adaptive benefits that the earliest stages of aggregation may provide in their own right.

In situ aggregation benefits: The shelter hypothesis. It seems plausible that the modern forms of aggregative multicellular structures evolved gradually. For example, in dictyostelids aggregation into fruiting bodies is hypothesised to have evolved through co-option of the process leading to macrocyst formation, a simpler form of aggregation present in many species (Kessin 2001). In general, aggregative structures presumably originated as slightly raised cell mounds which may have emerged in response to similar selective pressures. Simple biofilm associations have been reported to protect cells from abiotic stressors and biotic dangers (Oliveira et al. 2015; Trunk et al. 2018), and multicellularity has evolved in experimental populations of algae in response to predation pressure (Herron et al. 2019). Cells in the interior of an aggregate may be sheltered either by more distal cells (Smukalla et al. 2008; Kawaguchi et al. 2020) or by a layer of extracellular material produced at the group surface (Kirby, Garner and Levin 2012; Vega et al. 2014). The predatory nematode Caenorhabditis elegans has been observed to induce aggregation in D. discoideum (Kessin et al. 1996). This might be an indirect effect of increased competition for prey bacteria rather than an escape mechanism in direct response to $C$. elegans; regardless, these simple aggregates appear to protect cells from predation.

Larger groups may do a better job of protecting cells, and aggregation is a faster and more costeffective way than cell division to increase group size (Pentz et al. 2020, Tarnita, Taubes and Nowak 2013). Active aggregation into simple cell mounds may have increased protection from stress beyond what can be achieved within flat colonies or biofilms. Such simple shelter benefits are likely to have been one of the forces that selected for the earliest evolutionary stages of active aggregation.

There are inherent challenges in attempting to test the shelter hypothesis. For one thing, we don't have access to the non-aggregating ancestors of aggregating lineages. One could use non-aggregating mutants of extant genotypes as an alternative, although there is always the possibility for the mutations to create confounding effects. One could compare these mutants with wild-type strains and with other mutants that arrest development after early aggregation in order to investigate whether there are shelter benefits realized at that point that are not contingent on later, more complex morphological development, sporulation, or aggregate dispersal.

Influence of ecology on aggregative processes. Regardless of the selective forces that first favored primitive aggregation, the ecological factors involved in modern processes of aggregation differ across taxa. One natural isolate of D. mucoroides needs the presence of a specific fungus (Mucor hiemalis) in order to aggregate and develop into fruiting bodies (Ellison and Buss 1983). The protist Acrasis rosea generally requires a light:dark cycle in order to fruit (Reinhardt 1968). Aggregates of the 
myxobacterium Stigmatella aurantiaca form spore-bearing fruiting bodies only when exposed to light (Qualls, Stephens and White 1978). In M. xanthus, interaction with some other bacterial species can induce aggregation (Berleman and Kirby 2007), and the nutrient conditions triggering aggregation vary even among strains of the same species (Kraemer, Toups and Velicer 2010). These examples suggest that the evolution of aggregates from simple mounds into the range of structures seen today was likely influenced by varied and complex aspects of the specific ecologies of the organisms.

\section{Life in the aggregate}

Primitive aggregation, once evolved, potentiates further adaptive innovations. For example, aggregating lineages might evolve cell-surface adhesins to stabilize the aggregate or changes in aggregate size and morphology that increase shelter benefits or provide aggregate-dispersal benefits. However, identifying adaptive versus non-adaptive features of aggregates and of cellular behavior within aggregates can be challenging.

Active aggregate migration. While myxobacteria swarm cooperatively during vegetative growth (Muñoz-Dorado et al. 2016), aggregated Dictyostelium cells migrate together only during development in amorphous multicellular structures called slugs. Cells within a slug are not fully committed to continuing the developmental program until the slug begins to transform into a vertical fruiting body, at which point cells commit toward terminal differentiation into either stalk cells or spores (Kessin 2001). Slugs exhibit photo- and thermotaxis, and slug migration has been hypothesized to be an adaptive group behavior that improves positioning for subsequent fruiting body formation and spore dispersal (Kessin 2001; Bonner 2009). Slugs can migrate across some terrain more easily than single cells, and larger slugs are able to migrate over a larger area than smaller slugs (Foster et al. 2002). As discussed in chapter 8 , motile slugs may provide dispersal-related benefits. However, not all dictyostelids form motile slugs (Bonner 2003, Nanjundiah 2016, see chapter 8), and the evolutionary forces that led some genera to acquire this trait, whereas others did not, are worth investigating.

Cell fates within aggregates. One of the most evolutionarily fascinating features of many aggregative systems is that some cells die and some survive as spores. Joining an aggregate therefore can come with a high degree of fitness uncertainty. The extent of cell death may vary greatly across aggregative systems. In Myxococcus, only a minority of cells become heat-resistant spores under laboratory conditions, and extensive cell lysis occurs during aggregation, which has been suggested to be important for spore formation (Wireman and Dworkin 1977, O'Connor \& Zusman 1988, Lee et al 2012). In dictyostelids, $\sim 20-30 \%$ of the aggregated cells die and become part of a stalk while the remainder become viable spores elevated by the stalk (Kessin 2001). The mechanisms that determine whether aggregated dictyostelid cells die or survive as spores seem to be stochastic rather than deterministic, although developmental fate becomes more predictable if cells' physiological states are different at the onset of aggregation (Leach, Ashworth and Garrod 1973; Saran 1999; Azhar et al. 2001, Nanjundiah and Sathe, 2011 and 2013). Among the Copromyxa amoebae and the Acrasids, all cells remain alive during aggregative development. An initial Copromyxa cell founds a fruiting body by sporulating, and subsequently arriving cells travel to the top of the rising fruiting body and sporulate in turn (Spiegel 1978).

Differing cell fates during development have been viewed as a social "division of labor" that harms some individuals and benefits others in order to serve a greater social good (Zhang, Claessen and Rozen 2016). For social amoebae, it has been proposed that the extent of cell death in stalks may reflect an evolutionary tradeoff between the advantages of greater dispersal, which is hypothesized to be a result of increased cell death leading to taller stalks, versus a higher fraction of cells surviving to the next lifecycle (Kaushik and Nanjundiah 2003; Votaw and Ostrowski 2017). For the myxobacteria, in addition to hypotheses regarding fruiting body height and dispersal, it has also been suggested that components released by dying cells may be integrated into the spore coats of sporulating cells (Tientze et al. 1985), although this has not been clearly demonstrated. In all systems, there is still much to learn regarding the evolutionary forces that determine the extent of cell death during aggregative development.

Cells that remain outside the aggregate. Once aggregation has begun, cells must cooperate for development to proceed. However, in both M. xanthus and D. discoideum, not all cells respond to developmental signals by entering aggregates. Such non-aggregating cells are referred to as "peripheral rods" in M. xanthus (O'Connor \& Zusman 1991), whereas in D. discoideum they are called "loners" because individual cells remain isolated (Tarnita et al. 2015). D. discoideum loners avoid the prospect 
of dying in a stalk, and they may have an advantage if fresh nutrients become available while the rest of the population is involved in development because they can re-start vegetative growth and then divide more rapidly than aggregated cells, which eventually face an irreversible commitment to development (Dubravcic, Baalen, and Nizak 2014; Tarnita et al. 2015). It has been proposed that peripheral rods (O'Connor \& Zusman 1991; Kroos 2017) and loners (Tarnita et al. 2015, Rainey 2015) may represent bet-hedging strategies that optimize long-term fitness in fluctuating environments. Consistent with this hypothesis, the frequency of $D$. discoideum loners in a population is a heritable trait and therefore potentially subject to selection (Dubravcic, Baalen, and Nizak 2014; Rossine et al. 2020). Evolution experiments which manipulate nutrient levels over time could be performed to test such bet-hedging hypotheses in both myxobacteria and social amoebae.

Aggregate dispersal: The dispersal-benefit hypothesis. It has long been assumed that fruiting bodies enhance dispersal of spores and that this was a driving force in the emergence of these structures (Stanier 1942; Bonner 1982 and 2009; Kaiser 1993 and 2001). The dispersal-benefit hypothesis is attractive in light of the observation that aggregates can be induced by harsh environmental conditions (Tarnita, Taubes and Nowak 2013). Spores in taller fruiting bodies may have a greater likelihood of being picked up by an animal vector (smith, Queller and Strassmann 2014) or translocated by water or air. Does aggregative structure formation in fact increase average dispersal relative to the absence of development? And if average dispersal is increased, does it actually increase average cell fitness - by making it more likely for spores to experience a resource-rich environment - relative to sheltering in place? Studies that address either of these questions experimentally are few; most treatments of these questions involve speculation regarding potential implications of fruiting body architecture and the evolutionary purpose of cell death during aggregative development. For example, it has been hypothesized that taller stalks better facilitate dispersal (Bonner 2009; Sathe et al. 2010), and smith, Queller and Strassmann (2014) provide empirical evidence for this. It has also been proposed that utilizing dead cells to construct a stalk, as occurs in Dictyostelium, could be an adaptive innovation (Kaushik and Nanjundiah 2003) to produce taller, more robust stalks which might do more to facilitate dispersal than stalks built from living cells, as in the Acrasids (Brown 2011) and Copromyxa (Spiegel 1978), or stalks built from secreted extracellular components, as found in the Holozoa (Brown 2009).

One difficulty with the dispersal-benefit hypothesis is that it does not explain the morphological diversity of fruiting bodies seen across taxa. Beyond the core commonality of elongation away from a surface, fruiting bodies vary dramatically in size, shape, color, and complexity, not only across major taxa divisions (see Chapter 8) but also within monophyletic groups. For example, some myxobacterial species generate highly elevated and elaborate morphologies whereas others merely aggregate into simple, slightly heightened cell mounds (Fig. 1) (Dawid 2000). Such variation in fruiting body characteristics is hard to explain solely as a means to increase spore dispersal. Another difficulty is that mere aggregation into short, dense mounds may not suffice to provide dispersal-related benefits, and so the dispersal-benefit hypothesis may not explain the evolution of aggregation prior to the emergence of larger and more complex fruiting body morphologies.

Non-adaptive vs adaptive explanations of morphology and behavior. Laboratory evolution experiments in myxobacteria have shown that fruiting body morphology can evolve rapidly (Velicer et al. 1998; La Fortezza and Velicer 2021). For example, one recent study has found that the morphological evolution of fruiting bodies can be strongly influenced by social interactions between genotypes during aggregative development (La Fortezza and Velicer 2021). However, different evolutionary forces may be responsible for the morphologies of aggregates in natural populations.

While fruiting body morphology may have been influenced by selection, historical contingency and chance have likely also played a role in determining which lineages evolved taller and more complex fruiting bodies and which evolved smaller, simpler forms (Gould and Lewontin 1979; Travisano et al. 1995; Blount, Lenski and Losos 2018). Analogizing to organismal features, Lewontin and Gould (Gould and Lewontin 1979) famously remarked that the spandrels in Venice's San Marco Basilica can easily be viewed as having been designed to optimize display of the remarkable mosaics found on them, when in fact the spandrels are primarily aspects of architectural design which support the cathedral domes. Their point was that non-adaptive explanations for origins of organismal features should not be excluded without sufficient cause.

The relevance of indirect evolution in aggregative systems is highlighted by outcomes from several evolution experiments with myxobacteria that have recently been dubbed MyxoEEs (Rendueles and 
Velicer 2020). Forces other than direct selection have been shown to drive evolution of a range of traits, including extreme cheating phenotypes (Velicer, Kroos and Lenski 2000), social fitness inequalities between specific genotypes (referred to as cheating in Dictyostelium literature (e.g. Khare et al. 2009)), facultative social exploitation during development (Nair 2018), kin-discrimation phenotypes (Rendueles et al. 2015), colony-level morphology (Rendueles et al. 2020), quality as phage host (Freund et al. 2020), and even susceptibility to cheating (Schaal et al. 2021). Disentangling which features of aggregative systems evolved as adaptations and which did not remains a major challenge for future research.

\section{Life after aggregation}

There are striking contrasts among organisms in how cells behave when aggregates disband. In particular, the myxobacteria appear to differ from amoebae in the degree to which germinating spores and vegetative cells continue to interact and to cooperate. Whereas amoebae may live comparatively solitary lives except when they undergo aggregative development (Rubin et al. 2019), the myxobacteria remain social throughout their life cycle. For such systems that cooperate extensively during growth, aggregation may have density benefits that extend beyond the formation, survival and dispersal of spores (Ramsey and Dworkin 1968).

Both dictyostelids and myxobacteria initiate spore germination once environmental conditions near the aggregate become favorable, but while high density inhibits spore germination in $D$. discoideum (Cotter and Raper 1966; Ceccarini and Cohen 1967, Kessin 2001), M. xanthus spores germinate better at higher density (Pande et al. 2020). Such positive density-dependence of spore germination may therefore have promoted the evolution and maintenance of fruiting body formation in the myxobacteria but appears unlikely to have done so in the dictyostelids.

Freshly germinated cells face many potential challenges, including abiotic stressors such as acidity, difficulties in finding and consuming prey and encountering biotic enemies. Germinating in highdensity aggregates appears to help myxobacteria meet such challenges. M. xanthus is sensitive to even mild acidity but grows much better under acid stress at higher density (F Fiegna, S Pande, H Peitz, GJ Velicer, unpublished results). The rate at which groups of $M$. xanthus cells swarm across surfaces depends greatly on local cell density (Kaiser and Crosby 1983), which suggests that spores germinating in aggregates may be better than single spores at moving toward prey and away from dangers. There is indirect evidence that myxobacteria may sometimes benefit from being at high density during predation (Rosenberg, Keller and Dworkin 1977) and if this hypothesis is correct, spores germinating within fruiting bodies may have a predation advantage over non-aggregated spores. Additionally, like humans and other animals, bacteria frequently kill members of their own species (Granato et al. 2019); in the myxobacteria, higher density groups are more likely to survive these interactions (Rendueles, Amherd and Velicer 2015). In pairwise competitions between M. xanthus natural isolates, the genotype in the majority tends to have an advantage at killing or antagonizing the opponent. Spores will fare better in such combat by having germinated together rather than alone.

Dictyostelids are not known to engage in social motility, group hunting, lethal frequency-dependent warfare, or cooperative growth under stress. Germinated D. discoideum cells are asocial - moving, hunting, and reproducing as individuals and becoming cooperative only upon starvation. Advantages of aggregation in the dictyostelids may be limited to shelter and dispersal benefits, in contrast to the myxobacteria, whose cells appear to benefit from aggregate formation not only during development but also later during subsequent growth and collective migration. Such delayed indirect benefits of aggregation may have worked in concert with shelter and dispersal benefits in promoting its evolution in some systems.

\section{Genetic diversity within aggregative systems: The challenge of chimerism}

Because the types of aggregates examined here are not formed by a single dividing cell, they provide an opportunity for genetically distinct lineages to come together. While bacterial biofilms, for example, can contain many different genera (Nadell, Xavier and Foster 2009), even single-species aggregates often harbor genetic diversity (Filosa 1962; Buss 1982; Flowers et al. 2010; Sathe et al 2010; Kraemer and Velicer 2011; Nanjundiah 2016; Wielgoss et al. 2019). The potential for genetically distinct cells to cooperate, coalesce, and compete within cell aggregates creates the possibility for selection to operate at multiple levels - that of the single cell and that of the aggregate as a whole. In 
this way, whether aggregated groups are monoclonal or chimeric can determine the benefits and costs of actively aggregating with others (see Chapter 7).

Chimeric aggregates may be more frequent in nature than monoclonal ones. High local genetic diversity found in soil samples of both Myxococcus (Vos and Velicer 2006; Kraemer and Velicer 2011) and Dictyostelium (Fortunato et al. 2003; Ostrowski et al. 2015) indicates that genetically distinct strains can be close enough to each other that co-aggregation is at least a possibility. In M. xanthus, chimeric aggregates have indeed been isolated (Wielgoss et al. 2019). In this case, genotypes from the same fruiting bodies were very closely related, suggesting that they recently diverged from a common ancestor and remained in close enough proximity to co-aggregate. The cellular slime mold Acrasis rosea also forms chimeric fruiting bodies, although one strain tends to dominate, and the fruiting body shows features characteristic of those formed by that strain in monoculture (Reinhardt 1975). In dictyostelids, natural samples frequently yield distinct isolates capable of forming chimeric fruiting bodies (Filosa 1962; Buss 1982; Flowers et al. 2010; Sathe et al 2010; Nanjundiah 2016), although some $D$. discoideum fruiting bodies appear to be monoclonal (Gilbert et al. 2007; Gilbert, Queller and Strassmann 2009). Additionally, distinct genotypes of D. discoideum (Gilbert et al. 2007), D. giganteum (Kaushik, Katoch and Nanjundiah 2006), and D. mucoroides (Filosa 1962) have been observed to readily co-aggregate in the lab. Future comparisons of whole-genome sequences among co-aggregated cells will provide a finer-scale understanding of the degree of chimerism in natural aggregative systems (Wielgoss et al. 2019).

Negative effects of chimerism often dominate discussions of this phenomenon. Co-aggregation of distinct genotypes can reduce aggregate fitness, a phenomenon known as 'chimeric load' (Kraemer and Velicer 2011). Chimeric load can be observed even when all genotypes are equally proficient at development in monoculture. For example, chimeric M. xanthus fruiting bodies contain fewer spores when the mixed strains originated from different natural fruiting bodies (Mendes-Soares et al. 2014; Pande and Velicer 2018). Similarly, chimeric D. discoideum slugs migrate slower than clonal slugs of the same size (Foster et al. 2002). Such chimeric load might result from active antagonisms between genotypes or simply from dysfunctional divergence. A special form of chimeric load is 'cheating load' (Travisano and Velicer 2004; Fiegna and Velicer 2003), in which the presence of aggregation-defective cheaters in groups of aggregation-proficient cells reduces group-level spore productivity. Such negative effects of chimerism might be expected to lead to the evolution of mechanisms that limit it, a prospect that has received much attention (e.g. Strassmann et al. 2011). However, intrinsic patterns of cell growth in spatially structured habitats may sometimes suffice to greatly restrict chimerism in aggregating groups (smith, Strassmann and Queller 2016) even in the absence of kin-discriminatory traits.

In some cases, chimerism may increase the benefits of aggregation (Foster et al. 2002; Pineda-Krch and Lehtilä 2004; Pande and Velicer 2018). Strains with different phenotypes may be able to complement each other and increase overall group productivity (Bonner 1967). In Myxococcus, for example, natural isolates sampled from the same fruiting body produced fewer spores in monoclonal groups than when they underwent development together, suggesting synergistic interactions among these genotypes (Wielgoss et al. 2019). In another example, in pairwise mixes of three strains of $D$. giganteum, there was a significant tendency for one strain to be favored over the other in spore formation. However, when all three strains were mixed together, such asymmetry was significantly reduced, suggesting that there are higher-order interactions stabilizing the coexistence of different genotypes (Kaushik, Katoch and Nanjundiah 2006). As with other aspects of aggregate formation, the effect of chimerism is influenced by ecological conditions and by divergence across taxa.

\section{Conclusion}

Modern evolutionary theory originated mainly from studies of plants and animals that develop from a single cell. But in many taxa, there are single-celled organisms which have a very different life cycle, living at lower density under good conditions and then actively aggregating and forming a multicellular structure when conditions are bad (Fig. 2). The formation of these structures raises fascinating evolutionary questions. Why do cells aggregate even though this provides an opportunity for genetically different cells to take advantage? How have these complex processes emerged and changed over time? What is the relative strength of selection at different levels of these systems? 
By organizing themselves into multicellular aggregates, these organisms challenge our idea of biological individuality (Godfrey-Smith 2009; Ereshefsky and Pedroso 2012; Monte and Rainey 2014; West et al. 2015; Lidgard and Nyhart 2017). In general, obligately multicellular organisms reproduce via a single-cell bottleneck, so the group as a whole is the unit of selection (Lewontin 1970). For aggregating organisms, however, the unit of selection is context-dependent. Developmental aggregates can be seen as a survival bottleneck, but they are composed of cells which retain reproductive autonomy for the majority of the organismal life cycle.

Group-level selection may explain the emergence of some aspects of aggregative multicellular processes. However, the adaptive character, if any, of many traits specific to multicellular aggregates has not been clearly demonstrated. Even the most basic adaptive roles of the fruiting body structure itself require further investigation, and counterpoints to the dispersal-benefit hypothesis should be explored more extensively. The evolutionary causes of variation in fruiting body morphologies are even less well explained, although study of more recently evolved aggregative organisms such as Sorogena stoianovitchae (Lasek-Nesselquist 2001) may provide fresh insights distinct from those gained by study of more ancient systems.

Studying aggregating organisms is difficult because they can be challenging to isolate and to observe under natural conditions. To date, for example, the cellular slime mold Fonticula alba seems to have only been isolated once (Brown, Spiegel, and Silberman 2009). Guttulinopsis vulgaris (Brown et al. 2012b) and Sorodiplophrys stercorea (Tice 2016) are the only members of their supergroups (Rhizaria and Stramenopiles, respectively) known to show aggregative multicellularity. As a result, most studies of aggregative multicellularity have been conducted on dictyostelids and myxobacteria, and on very few species from those two groups. Development of new isolation methods may reveal a broader diversity of aggregative microbial systems in nature and so provide a more thorough understanding of the evolutionary forces that can shape aggregative multicellularity.

\section{Box 1 - Aggregative behaviors}

Here we use the terms aggregative behavior and aggregation to refer to those processes in which single units come together to form or join a group, which represents a higher-level unit of organization and potentially of selection. Aggregation occurs not only among autonomously reproducing cells (the focus of this chapter), but also among cells within animal bodies and among multicellular animals. Examples of cellular aggregation within animal bodies include aggregation among platelet cells during wound healing (Savage, Almus-Jacob and Ruggeri 1998), germ-line and mesenchymal cells during embryogenesis (Savage and Danilchik 1993; O’Shea 1987), single sponge cells after dissociation (Wilson 1907; Padua et al. 2016), and cancer cells in the bloodstream during metastasis (Aceto et al. 2014). Animal groups such as herds, flocks, and schools also result from aggregative behavior. Many of the proposed advantages and disadvantages of aggregating into groups are similar for cells and animals, including protection from abiotic stresses and predation and enhanced migration (Krause and Ruxton 2002; West et al. 2015). Although very different from group formation, plants and fungi exhibit coming-together-like behavior in the act of fusing to form chimeric organisms composed of two or more distinct genotypes (Buss 1982). For example, hyphae of genetically distinct fungi can fuse and form chimeric fruiting bodies (Glass et al. 2004) and plant chimeras can emerge from genetically distinct individuals that merge during growth (Frank and Chitwood 2016). 


\section{Bibliography}

Aceto, N., Aditya Bardia, David T. Miyamoto, Maria C. Donaldson, Ben S. Wittner, Joel A. Spencer, $\mathrm{Min} \mathrm{Yu}$, et al. 2014. "Circulating tumor cell clusters are oligoclonal precursors of breast cancer metastasis." Cell 158: 1110-22. https://doi.org/10.1016/j.cell.2014.07.013.

Angel, Juan A. Arias Del, Vidyanand Nanjundiah, Mariana Benítez, and Stuart A. Newman. 2020. "Interplay of mesoscale physics and agent-like behaviors in the parallel evolution of aggregative multicellularity". EvoDevo 11: 21

Azhar, M, P. K. Kennady, G. Pande, M. Espiritu, W. Holloman, D. Brazill, R. H. Gomer, and V. Nanjundiah. 2001. "Cell cycle phase, cellular Ca2+ and development in Dictyostelium discoideum." The International Journal of Developmental Biology 45: 405-14.

Berleman, James E., and John R. Kirby. 2007. "Multicellular development in Myxococcus xanthus is stimulated by predator-prey interactions." Journal of Bacteriology 189: 5675-82. https://doi.org/10.1128/jb.00544-07.

Blanton, R. L., and L. S. Olive. 1983. "Ultrastructure of aerial stalk formation by the ciliated protozoan Sorogena stoianovitchae." Protoplasma 116: 125-35. https://doi.org/10.1007/bf01279829.

Blount, Z. D., R. E. Lenski, and J. B. Losos. 2018. "Contingency and determinism in evolution: Replaying life's tape.” Science 362: eaam5979. https://doi.org/10.1126/science.aam5979.

Bonner, J. T. 1967. The cellular slime molds. (2nd. ed). Princeton, KJ: Princeton University Press.

Bonner, J. T. 1982. "Evolutionary strategies and developmental constraints in the cellular slime molds." The American Naturalist 119, 530-52.

Bonner, J. T. 2003. "Evolution of development in the cellular slime molds." Evolution \& Development 5: 305-13. https://doi.org/10.1046/j.1525-142x.2003.03037.x.

Bonner, J. T. 2009. The social amoebae: The biology of cellular slime molds. Princeton; Oxford: Princeton University Press.

Branda, S. S., J. E. Gonzalez-Pastor, S. Ben-Yehuda, R. Losick, and R. Kolter. 2001. "Fruiting body formation by Bacillus subtilis." Proceedings of the National Academy of Sciences U.S.A. 98: 1162126. https://doi.org/10.1073/pnas.191384198.

Brown, Matthew W., F. W., Spiegel, and Jeffrey D. Silberman. 2009."Phylogeny of the "forgotten" cellular slime mold, Fonticula alba, reveals a key evolutionary branch within opisthokonta." Molecular Biology \& Evolution 26: 2699-2709.

Brown, Matthew W., Jeffrey D. Silberman, and F. W., Spiegel. 2012. "A contemporary evaluation of the acrasids (Acrasidae, Heterolobosea, Excavata)." European Journal of Protistology 48: 103-123.

Brown, Matthew W., Martin Kolisko, Jeffrey D. Silberman, and Andrew J. Roger. 2012b. "Aggregative multicellularity evolved independently in the eukaryotic supergroup Rhizaria." Current Biology 22: 1123-27. https://doi.org/10.1016/j.cub.2012.04.021.

Brown, Matthew W., and Jeffrey D. Silberman. 2013. "The non-dictyostelid sorocarpic amoebae." in Dictyostelids, evolution, genomics and cell biology. 219-42. https://doi.org/10.1007/978-3-642-384875_12.

Buss, L. W. 1982. "Somatic cell parasitism and the evolution of somatic tissue compatibility." Proceedings of the National Academy of Sciences U.S.A. 79: 5337-41. https://doi.org/10.1073/pnas.79.17.5337.

Ceccarini, Costante, and Arthur Cohen. 1967. "Germination inhibitor from the cellular slime mould Dictyostelium discoideum.” Nature 214: 1345-46. https://doi.org/10.1038/2141345a0. 
Claessen, Dennis, Daniel E. Rozen, Oscar P. Kuipers, Lotte Søgaard-Andersen, and Gilles P. van Wezel. 2014. "Bacterial solutions to multicellularity: A tale of biofilms, filaments and fruiting bodies." Nature Reviews Microbiology 12: 115-24. https://doi.org/10.1038/nrmicro3178.

Cotter, D. A., and K. B. Raper. 1966. "Spore germination in Dictyostelium discoideum." Proceedings of the National Academy of Sciences U.S.A. 56: 880-87. https://doi.org/10.1073/pnas.56.3.880.

Dawid, W. 2000. "Biology and global distribution of myxobacteria in soils." FEMS Microbiology Reviews 24: 403-27. https://doi.org/10.1111/j.1574-6976.2000.tb00548.x.

Du, Qingyou, Yoshinori Kawabe, Christina Schilde, Zhi-hui Chen, and Pauline Schaap. 2015. "The evolution of aggregative multicellularity and cell-cell communication in the Dictyostelia." Journal of Molecular Biology 427: 3722-33. https://doi.org/10.1016/j.jmb.2015.08.008.

Dubravcic, Darja, Minus van Baalen, and Clément Nizak. 2014. "An evolutionarily significant unicellular strategy in response to starvation in Dictyostelium social amoebae." F1000Research 3: 133. https://doi.org/10.12688/f1000research.4218.2.

Dykstra, Michael J., and Lindsay S. Olive. 1975. "Sorodiplophrys: An unusual sorocarp-producing protist." Mycologia 67: 873. https://doi.org/10.2307/3758346.

Ellison, Aaron M., and Leo W. Buss. 1983. "A naturally occurring developmental synergism between the cellular slime mold, Dictyostelium mucoroides and the fungus, Mucor hiemalis." American Journal of Botany 70 (2): 298-302. https://doi.org/10.1002/j.1537-2197.1983.tb07870.x.

Ereshefsky, Marc, and Makmiller Pedroso. 2012. "Biological individuality: The case of biofilms." Biology \& Philosophy 28: 331-49. https://doi.org/10.1007/s10539-012-9340-4.

Fiegna, Francesca, and Gregory J. Velicer. 2003. "Competitive fates of bacterial social parasites: Persistence and self-induced extinction of Myxococcus xanthus cheaters." Proceedings of the Royal Society B: Biological Sciences 270: 1527-34. https://doi.org/10.1098/rspb.2003.2387.

Fiegna, Francesca, and Gregory J. Velicer. 2005. "Exploitative and hierarchical antagonism in a cooperative bacterium." PLoS Biology 3: e370. https://doi.org/10.1371/journal.pbio.0030370.

Filosa, M. F. 1962. "Heterocytosis in cellular slime molds." The American Naturalist 96: 79-91. https://doi.org/10.1086/282209.

Flärdh, Klas, David M. Richards, Antje M. Hempel, Martin Howard, and Mark J Buttner. 2012. "Regulation of apical growth and hyphal branching in Streptomyces." Current Opinion in Microbiology 15: 737-43. https://doi.org/10.1016/j.mib.2012.10.012.

Flores, Enrique, and Antonia Herrero. 2010. "Compartmentalized function through cell differentiation in filamentous Cyanobacteria." Nature Reviews Microbiology 8: $39-50$. https://doi.org/10.1038/nrmicro2242.

Flowers, Jonathan M., Si I. Li, Angela Stathos, Gerda Saxer, Elizabeth A. Ostrowski, David C. Queller, Joan E. Strassmann, and Michael D. Purugganan. 2010. "Variation, sex, and social cooperation: Molecular population genetics of the social amoeba Dictyostelium discoideum." PLoS Genetics 6: e1001013. https://doi.org/10.1371/journal.pgen.1001013.

Fortunato, A., J. E. Strassmann, L. Santorelli, and D. C. Queller. 2003. "Co-occurrence in nature of different clones of the social amoeba, Dictyostelium discoideum." Molecular Ecology 12: 1031-38. https://doi.org/10.1046/j.1365-294x.2003.01792.x.

Foster, Kevin R., Angelo Fortunato, Joan E. Strassmann, and David C. Queller. 2002. "The costs and benefits of being a chimera." Proceedings of the Royal Society of London. Series B: Biological Sciences 269: 2357-62. https://doi.org/10.1098/rspb.2002.2163.

Frank, Margaret H., and Daniel H. Chitwood. 2016. "Plant chimeras: The good, the bad, and the 'Bizzaria."” Developmental Biology 419: 41-53. https://doi.org/10.1016/j.ydbio.2016.07.003. 
Freund L, Vasse M, Velicer GJ. 2021 Hidden paths to endless forms most wonderful: parasite-blind diversification of host quality. Proc. R. Soc. B 20210456. https://doi.org/10.1098/rspb.2021.0456

Gilbert, Owen M., Kevin R. Foster, Natasha J. Mehdiabadi, Joan E. Strassmann, and David C. Queller. 2007. "High relatedness maintains multicellular cooperation in a social amoeba by controlling cheater mutants." Proceedings of the National Academy of Sciences U.S.A. 104: 8913-17. https://doi.org/10.1073/pnas.0702723104.

Gilbert, Owen M., David C. Queller, and Joan E. Strassmann. 2009. "Discovery of a large clonal patch of a social amoeba: Implications for social evolution." Molecular Ecology 18: 1273-81. https://doi.org/10.1111/j.1365-294x.2009.04108.x.

Glass, N. Louise, Carolyn Rasmussen, M. Gabriela Roca, and Nick D. Read. 2004. "Hyphal homing, fusion and mycelial interconnectedness." Trends in Microbiology 12: 135-41. https://doi.org/10.1016/j.tim.2004.01.007.

Godfrey-Smith, Peter. 2009. Darwinian populations and natural selection. Oxford: Oxford University Press.

Gould, S. J., and R. C. Lewontin. 1979. "The spandrels of San Marco and the Panglossian paradigm: A critique of the adaptationist programme." Proceedings of the Royal Society of London. Series B. Biological Sciences 205: 581-98. https://doi.org/10.1098/rspb.1979.0086.

Granato, Elisa T., Thomas A. Meiller-Legrand, and Kevin R. Foster. 2019. "The evolution and ecology of bacterial warfare." Current Biology 29: R521-37. https://doi.org/10.1016/j.cub.2019.04.024.

Grilione, P. L., and J. Pangborn. 1975. "Scanning electron microscopy of fruiting body formation by myxobacteria." Journal of Bacteriology 124: 1558-65. https://doi.org/10.1128/jb.124.3.15581565.1975.

Herron, Matthew D., Joshua M. Borin, Jacob C. Boswell, Jillian Walker, I-Chen Kimberly Chen, Charles A. Knox, Margrethe Boyd, Frank Rosenzweig, and William C. Ratcliff. 2019. "De novo origins of multicellularity in response to predation." Scientific Reports 9: 2328. https://doi.org/10.1038/s41598019-39558-8.

Houry, A., R. Briandet, S. Aymerich, and M. Gohar, 2010. "Involvement of motility and flagella in Bacillus cereus biofilm formation." Microbiology 156, 1009-1018.

Kaiser, Dale, and Cathy Crosby. 1983. "Cell movement and its coordination in swarms of Myxococcus xanthus." Cell Motility 3: 227-45. https://doi.org/10.1002/cm.970030304.

Kaiser, D. 1993. "Roland Thaxter's legacy and the origins of multicellular development." Genetics 135 (2): 249-54.

Kaiser, Dale. 2001. "Building a multicellular organism." Genetics 35: 103-23. https://doi.org/10.1146/annurev.genet.35.102401.090145.

Kaplan, Heidi B., and Lynda Plamann. 1996. "A Myxococcus xanthus cell density-sensing system required for multicellular development." FEMS Microbiology Letters 139: 89-95. https://doi.org/10.1111/j.1574-6968.1996.tb08185.x.

Kaushik, Sonia, and Vidyanand Nanjundiah. 2003. "Evolutionary questions raised by cellular slime mould development" Proceedings of the Indian National Sciences Academy B69: 825-852

Kaushik, Sonia, Bandhana Katoch, and Vidyanand Nanjundiah. 2006. "Social behaviour in genetically heterogeneous groups of Dictyostelium giganteum." Behavioral Ecology and Sociobiology 59: 521-30. https://doi.org/10.1007/s00265-005-0077-9.

Kawabe, Yoshinori, Qingyou Du, Christina Schilde, and Pauline Schaap. 2019. "Evolution of multicellularity in Dictyostelia." International Journal of Developmental Biology 63: 359-69. https://doi.org/10.1387/ijdb.190108ps. 
Kawaguchi, Yuko, Mio Shibuya, Iori Kinoshita, Jun Yatabe, Issay Narumi, Hiromi Shibata, Risako Hayashi, et al. 2020. "DNA damage and survival time course of Deinococcal cell pellets during 3 years of exposure to outer space." Frontiers in Microbiology 11: 2050. https://doi.org/10.3389/fmicb.2020.02050.

Kessin, R. H., G. G. Gundersen, V. Zaydfudim, and M. Grimson. 1996. "How cellular slime molds evade nematodes." Proceedings of the National Academy of Sciences U.S.A. 93: 4857-61. https://doi.org/10.1073/pnas.93.10.4857.

Kessin, R. 2001. Dictyostelium: Evolution, Cell Biology, and the Development of Multicellularity. Cambridge: Cambridge University Press. doi:10.1017/CBO9780511525315

Khare, Anupama, Lorenzo A. Santorelli, Joan E. Strassmann, David C. Queller, Adam Kuspa, and Gad Shaulsky. 2009. "Cheater-resistance is not futile." Nature 461: 980-82. https://doi.org/10.1038/nature08472.

Kirby, Amy E., Kimberly Garner, and Bruce R. Levin. 2012. "The relative contributions of physical structure and cell density to the antibiotic susceptibility of bacteria in biofilms." Antimicrobial Agents and Chemotherapy 56: 2967-75. https://doi.org/10.1128/aac.06480-11.

Kraemer, Susanne A., Melissa A. Toups, and Gregory J. Velicer. 2010. "Natural variation in developmental life-history traits of the bacterium Myxococcus xanthus." FEMS Microbiology Ecology, 73: 226-233, https://doi.org/10.1111/j.1574-6941.2010.00888.x

Kraemer, Susanne A., and Gregory J. Velicer. 2011. "Endemic social diversity within natural kin groups of a cooperative bacterium." Proceedings of the National Academy of Sciences of the U.S.A.. 108 (Supplement 2): 10823-30. https://doi.org/10.1073/pnas.1100307108.

Krause, Jens, and Graeme D. Ruxton. 2002. Living in groups. Oxford: Oxford University Press.

Kroos, Lee. 2017. "Highly signal-responsive gene regulatory network governing myxococcus development." Trends in Genetics 33: 3-15. https://doi.org/10.1016/j.tig.2016.10.006.

La Fortezza, M. and G. J. Velicer. 2021. "Social selection within aggregative multicellular development drives morphological evolution." bioRxiv 2021.05.12.443771; doi: https://doi.org/10.1101/2021.05.12.443771

Lasek-Nesselquist, E., and L. A. Katz. 2001. "Phylogenetic position of Sorogena stoianovitchae and relationships within the class Colpodea (Ciliophora) based on SSU rDNA sequences". Journal Eukaryotic Microbiology 48: 604-607.

Leach, C. K., J. M. Ashworth, and D. R. Garrod. 1973. "Cell sorting out during the differentiation of mixtures of metabolically distinct populations of Dictyostelium discoideum." Journal of Embryology and Experimental Morphology 29 (3): 647-61.

Lee B., Holkenbrink C., Treuner-Lange A., Higgs P. I. (2012). Myxococcus xanthus developmental cell fate: heterogeneous accumulation of developmental regulatory proteins and reexamination of the role of MazF in developmental lysis. J. Bacteriol. 194: 3058-3068

Lewontin, R. C. 1970. "The Units of Selection." Annual Review of Ecology and Systematics 1: 1-18. https://doi.org/10.1146/annurev.es.01.110170.000245.

Li, Yinuo, Hong Sun, Xiaoyuan Ma, Ann Lu, Renate Lux, David Zusman, and Wenyuan Shi. 2003. "Extracellular polysaccharides mediate pilus retraction during social motility of Myxococcus xanthus." Proceedings of the National Academy of Sciences U.S.A. 100: 5443-48. https://doi.org/10.1073/pnas.0836639100.

Lidgard, Scott, and Lynn K. Nyhart. 2019. "Biological individuality: Integrating scientific, philosophical, and historical perspectives." https://doi.org/10.7208/9780226446592-002. 
Manhes, Pauline, and Gregory J. Velicer. 2011. "Experimental evolution of selfish policing in social bacteria." Proceedings of the National Academy of Sciences U.S.A. 108: 8357-62. https://doi.org/10.1073/pnas.1014695108.

Medina, James M., P.M. Shreenidhi, Tyler J. Larsen, David C. Queller, and Joan E. Strassmann. 2019. "Cooperation and conflict in the social amoeba Dictyostelium discoideum." International Journal of Developmental Biology 63: 371-82. https://doi.org/10.1387/ijdb.190158jm.

Meena, N. P. \& Kimmel, A. R. 2017. "Chemotactic network responses to live bacteria show independence of phagocytosis from chemoreceptor sensing." Elife 6, e24627. https://doi.org/10.7554/elife.24627

Mendes-Soares, H., I. C. K. Chen, K. Fitzpatrick, and G. J. Velicer. 2014. "Chimaeric load among sympatric social bacteria increases with genotype richness." Proceedings of the Royal Society B: Biological Sciences 281: 20140285-20140285. https://doi.org/10.1098/rspb.2014.0285.

Monte, Silvia De, and Paul B Rainey. 2014. "Nascent multicellular life and the emergence of individuality." Journal of Biosciences 39: 237-48. https://doi.org/10.1007/s12038-014-9420-5.

Muñoz-Dorado, José, Francisco J. Marcos-Torres, Elena García-Bravo, Aurelio Moraleda-Muñoz, and Juana Pérez. 2016. "Myxobacteria: Moving, killing, feeding, and surviving together." Frontiers in Microbiology 7: 2475-18. https://doi.org/10.3389/fmicb.2016.00781.

Nadell, Carey D., Joao B. Xavier, and Kevin R. Foster. 2009. “The sociobiology of biofilms." FEMS Microbiology Reviews 33: 206-24. https://doi.org/10.1111/j.1574-6976.2008.00150.x.

Nair, Ramith R., Francesca Fiegna, and Gregory J. Velicer. 2018. "Indirect evolution of social fitness inequalities and facultative social exploitation." Proceedings of the Royal Society B: Biological Sciences 285: 20180054. https://doi.org/10.1098/rspb.2018.0054.

Nanjundiah, Vidyanand, and Santosh Sathe. 2011. "Social selection and the evolution of cooperative groups: The example of the cellular slime moulds." Integrative Biology 3: 329-42. https://doi.org/10.1039/c0ib00115e.

Nanjundiah, Vidyanand, and Santosh Sathe. 2013. "Dictyostelids: Evolution, genomics and cell Biology," 193-217. https://doi.org/10.1007/978-3-642-38487-5_11.

Nanjundiah, Vidyanand. 2016. Cellular slime molds and aggregative multicellularity. in: "Multicellularity: Origins and evolution." Cambridge, MA: The MIT Press

Nudleman, Eric, Daniel Wall, and Dale Kaiser. 2005. "Cell-to-cell transfer of bacterial outer membrane lipoproteins." Science 309: 125-27. https://doi.org/10.1126/science.1112440.

O'Connor, K. A., and D. R. Zusman. 1988. "Reexamination of the role of autolysis in the development of Myxococcus xanthus." Journal of Bacteriology 170: 4103-12. https://doi.org/10.1128/jb.170.9.41034112.1988 .

O'Connor, K. A., and D. R. Zusman. 1991. "Development in Myxococcus xanthus involves differentiation into two cell types, peripheral rods and spores." Journal of Bacteriology 173: 3318-33. https://doi.org/10.1128/jb.173.11.3318-3333.1991.

O’Shea, K. S. 1987. "Differential deposition of basement membrane components during formation of the caudal neural tube in the mouse embryo." Development 99: 509-19.

Oliveira, Nuno M., Nuno M. Oliveria, Esteban Martinez-Garcia, Joao Xavier, William M Durham, Roberto Kolter, Wook Kim, and Kevin R. Foster. 2015. "Biofilm formation as a response to ecological competition.” PLoS Biology 13: e1002191. https://doi.org/10.1371/journal.pbio.1002191.

Ostrowski, Elizabeth A., Yufeng Shen, Xiangjun Tian, Richard Sucgang, Huaiyang Jiang, Jiaxin Qu, Mariko Katoh-Kurasawa, et al. 2015. "Genomic signatures of cooperation and conflict in the social amoeba.” Current Biology 25: 1661-65. https://doi.org/10.1016/j.cub.2015.04.059. 
Padua, André, Pedro Leocorny, Márcio Reis Custódio, and Michelle Klautau. 2016. "Fragmentation, fusion, and genetic homogeneity in a calcareous sponge (Porifera, Calcarea)." Journal of Experimental Zoology Part A: Ecological Genetics and Physiology 325: 294-303. https://doi.org/10.1002/jez.2017.

Pan, Pauline, E. M. Hall, and J. T. Bonner. 1972. "Folic acid as second chemotactic substance in the cellular slime moulds." Nature New Biology 237: 181-82. https://doi.org/10.1038/newbio237181a0.

Pande, Samay, and Gregory J. Velicer. 2018. "Chimeric synergy in natural social groups of a cooperative microbe." Current Biology 28: 262-267.e3. https://doi.org/10.1016/j.cub.2017.11.043.

Pande, Samay, Pau Pérez Escriva, Yuen-Tsu Nicco Yu, Uwe Sauer, and Gregory J. Velicer. 2020. "Cooperation and cheating among germinating spores." Current Biology 30: 4745-4752.e4. https://doi.org/10.1016/j.cub.2020.08.091.

Pentz, Jennifer T., Pedro Márquez-Zacarías, G. Ozan Bozdag, Anthony Burnetti, Peter J. Yunker, Eric Libby, and William C. Ratcliff. 2020. "Ecological advantages and evolutionary limitations of aggregative multicellular development.” Current Biology. https://doi.org/10.1016/j.cub.2020.08.006.

Pineda-Krch, M., and K. Lehtilä. 2004. "Costs and benefits of genetic heterogeneity within organisms." Journal of Evolutionary Biology 17: 1167-77. https://doi.org/10.1111/j.1420-9101.2004.00808.x.

Qualls, G. T., K. Stephens, and D. White. 1978. "Light-stimulated morphogenesis in the fruiting myxobacterium Stigmatella aurantiaca." Science 201: 444-45. https://doi.org/10.1126/science.96528.

Rainey, Paul B. 2015. "Precarious development: The uncertain social life of cellular slime molds." Proceedings of the National Academy of Sciences U.S.A. 112: 2639-40. https://doi.org/10.1073/pnas.1500708112.

Ramsey, W. Scott, and Martin Dworkin. 1968. "Microcyst germination in Myxococcus xanthus." Journal of Bacteriology 95: 2249-57. https://doi.org/10.1128/jb.95.6.2249-2257.1968.

Reinhardt, D. J. 1968. "The effects of light on the development of the cellular slime mold acrasis rosea". American Journal of Botany 55: 77-86.

Rendueles, Olaya, Michaela Amherd, and Gregory J. Velicer. 2015. "Positively frequency-dependent interference competition maintains diversity and pervades a natural population of cooperative microbes." Current Biology 25: 1673-81. https://doi.org/10.1016/j.cub.2015.04.057.

Rendueles, Olaya, Peter C Zee, Iris Dinkelacker, Michaela Amherd, Sébastien Wielgoss, and Gregory J Velicer. 2015. "Rapid and widespread de novo evolution of kin discrimination." Proceedings of the National Academy of Sciences U.S.A. 112: 9076-81. https://doi.org/10.1073/pnas.1502251112.

Rendueles, Olaya, and Gregory J. Velicer. 2020. "Hidden paths to endless forms most wonderful: Complexity of bacterial motility shapes diversification of latent phenotypes." BMC Evolutionary Biology 20: 145 . https://doi.org/10.1186/s12862-020-01707-3.

Rivera-Yoshida, Natsuko, Alejandra Hernández-Terán, Ana E. Escalante, and Mariana Benítez. 2019. "Laboratory biases hinder Eco-Evo-Devo integration: Hints from the microbial world." Journal of Experimental Zoology Part B: Molecular and Developmental Evolution 334: 14-24. https://doi.org/10.1002/jez.b.22917.

Rosenberg, E., K. H. Keller, and M Dworkin. 1977. "Cell density-dependent growth of Myxococcus xanthus on casein." Journal of Bacteriology 129: 770-77. https://doi.org/10.1128/jb.129.2.770777.1977.

Rossine, Fernando W., Ricardo Martinez-Garcia, Allyson E. Sgro, Thomas Gregor, and Corina E. Tarnita. 2020. "Eco-Evolutionary significance of "Loners." PLoS Biology 18: e3000642. https://doi.org/10.1371/journal.pbio.3000642. 
Rubin M, Miller AD, Katoh-Kurasawa M, Dinh C, Kuspa A, Shaulsky G. 2019. Cooperative predation in the social amoebae Dictyostelium discoideum. PLoS ONE 14(1): e0209438. https:// doi.org/10.1371/journal.pone.0209438

Saran, Shweta. 1999. "Calcium levels during cell cycle correlate with cell fate of Dictyostelium discoideum." Cell Biology International 23: 399-405. https://doi.org/10.1006/cbir.1999.0379.

Sathe, Santosh, Sonia Kaushik, Albert Lalremruata, Ramesh K. Aggarwal, James C. Cavender, and Vidyanand Nanjundiah. 2010. "Genetic heterogeneity in wild isolates of cellular slime mold social groups.” Microbial Ecology 60: 137-48. https://doi.org/10.1007/s00248-010-9635-4.

Savage, Robert M., and Michael V. Danilchik. 1993. "Dynamics of germ plasm localization and its inhibition by ultraviolet irradiation in early cleavage Xenopus embryos." Developmental Biology 157: 371-82. https://doi.org/10.1006/dbio.1993.1142.

Savage, Brian, Fanny Almus-Jacobs, and Zaverio M. Ruggeri. 1998. "Specific synergy of multiple substrate-receptor interactions in platelet thrombus formation under flow." Cell 94: 657-66. https://doi.org/10.1016/s0092-8674(00)81607-4.

Schaal, K. A.,Y.-T. N. Yu, M. Vasse and G. J. Velicer. 2021. “Allopatric divergence limits cheating range and alters genetic requirements for a cooperative trait". bioRxiv 2021.01.07.425765. doi:10.1101/2021.01.07.425765.

Smith, Daniel R., Manuel Maestre-Reyna, Gloria Lee, Harry Gerard, Andrew H.-J. Wang, and Paula I. Watnick. 2015. "In situ proteolysis of the vibrio cholerae matrix protein RbmA promotes biofilm recruitment." Proceedings of the National Academy of Sciences U.S.A. 112: 10491-96. https://doi.org/10.1073/pnas.1512424112.

smith, jeff, David C. Queller, and Joan E. Strassmann. 2014. "Fruiting bodies of the social amoeba Dictyostelium discoideum increase spore transport by Drosophila." BMC Evolutionary Biology 14 (1): 105. https://doi.org/10.1186/1471-2148-14-105.

smith, jeff, Joan E. Strassmann, and David C. Queller. 2016. "Fine-scale spatial ecology drives kin selection relatedness among cooperating amoebae." Evolution 70: 848-59. https://doi.org/10.1111/evo.12895.

Smukalla, Scott, Marina Caldara, Nathalie Pochet, Anne Beauvais, Stephanie Guadagnini, Chen Yan, Marcelo D. Vinces, et al. 2008. "FLO1 is a variable green beard gene that drives biofilm-like cooperation in budding yeast." Cell 135: 726-37. https://doi.org/10.1016/j.cell.2008.09.037.

Spiegel, Frederick W., L. S. Olive. "New evidence for the validity of Copromyxa protea". 1978. Mycologia 70: 843-847

Sproer, C., H. Reichenbach, and E. Stackebrandt. 1999. "The correlation between morphological and phylogenetic classification of myxobacteria." International Journal of Systematic Bacteriology 49: 1255-62. https://doi.org/10.1099/00207713-49-3-1255.

Stanier, R. Y. 1942. "The Cytophaga group: a contribution to the biology of myxobacteria." Bacteriological Reviews 6: 143-96.

Stenhouse, Fay O., and Keith L. Williams. 1977. "Patterning in Dictyostelium discoideum: The proportions of the three differentiated cell types (spore, stalk, and basal disk) in the fruiting body." Developmental Biology 59: 140-52. https://doi.org/10.1016/0012-1606(77)90249-4.

Strassmann, Joan E., Yong Zhu, and David C. Queller. 2000. "Altruism and social cheating in the social amoeba Dictyostelium discoideum." Nature 408: 965-67. https://doi.org/10.1038/35050087.

Strassmann, J. E., and D. C. Queller. 2011. "Evolution of cooperation and control of cheating in a social microbe." Proceedings of the National Academy of Sciences U.S.A. 108: 10855-62. https://doi.org/10.1073/pnas.1102451108. 
Sugimoto, Hiroki, and Hiroshi Endoh. 2006. "Analysis of fruiting body development in the aggregative ciliate Sorogena stoianovitchae (Ciliophora, Colpodea)." The Journal of Eukaryotic Microbiology 53: 96-102. https://doi.org/10.1111/j.1550-7408.2005.00077.x.

Swanson, Andrew R., Eduardo M. Vadell, and James C. Cavender. 1999. "Global distribution of forest soil Dictyostelids." Journal of Biogeography 26: 133-48. https://doi.org/10.1046/j.13652699.1999.00250.x.

Tarnita, Corina E, Clifford H Taubes, and Martin A Nowak. 2013. "Evolutionary construction by staying together and coming together." Journal of Theoretical Biology 320: 10-22. https://doi.org/10.1016/j.jtbi.2012.11.022.

Tarnita, Corina E., Alex Washburne, Ricardo Martinez-Garcia, Allyson E. Sgro, and Simon A. Levin. 2015. "Fitness tradeoffs between spores and nonaggregating cells can explain the coexistence of diverse genotypes in cellular slime molds." Proceedings of the National Academy of Sciences U.S.A. 112:277681. https://doi.org/10.1073/pnas.1424242112.

Teintze, M., R. Thomas, T. Furuichi, M. Inouye, and S. Inouye. 1985. "Two homologous genes coding for spore-specific proteins are expressed at different times during development of Myxососсиs xanthus." Journal of Bacteriology 163 (1): 121-25. https://doi.org/10.1128/jb.163.1.121-125.1985.

Thaxter, R. "On the Myxobacteriaceæ, a New Order of Schizomycetes".1892. Botanical Gazette 17, 389-406.

Tice, Alexander K., Jeffrey D. Silberman, Austin C. Walthall, Khoa N. D. Le, Frederick W. Spiegel, Matthew W. Brown. 2016. "Sorodiplophrys stercorea: Another novel lineage of sorocarpic multicellularity.” Journal of Eukaryotic Microbiology 63: 623-628.

Travisano, M., J. Mongold, A. Bennett, and R. Lenski. 1995. "Experimental tests of the roles of adaptation, chance, and history in evolution." Science 267: 87-90. https://doi.org/10.1126/science.7809610.

Travisano, M. and G. J. Velicer. 2004. "Strategies of microbial cheater control.” Trends in Microbiology 12: $72-78$

Trunk, Thomas, Hawzeen S. Khalil, Jack C. Leo, and Bacterial Cell Surface Group Norway Section for Genetics and Evolutionary Biology, Department of Biosciences, University of Oslo, Oslo,. 2018. "Bacterial autoaggregation." AIMS Microbiology 4: 140-64. https://doi.org/10.3934/microbiol.2018.1.140.

Vega, Nicole M., and Jeff Gore. 2014. "Collective antibiotic resistance: Mechanisms and implications." Current Opinion in Microbiology 21: 28-34. https://doi.org/10.1016/j.mib.2014.09.003.

Velicer, Gregory J., Lee Kroos, and Richard E. Lenski. 1998. "Loss of social behaviors by Myxococcus xanthus during evolution in an unstructured habitat." Proceedings of the National Academy of Sciences U.S.A. 95: 12376-80. https://doi.org/10.1073/pnas.95.21.12376.

Velicer, G. J., L. Kroos, and R. E. Lenski. 2000. "Developmental cheating in the social bacterium Myxococcus xanthus." Nature 404: 598-601

Velicer, Gregory J., and Michiel Vos. 2009. "Sociobiology of the myxobacteria." Annual Review of Microbiology 63: 599-623. https://doi.org/10.1146/annurev.micro.091208.073158.

Verstrepen, Kevin J., and Frans M. Klis. 2006. "Flocculation, adhesion and biofilm formation in yeasts." Molecular Microbiology 60: 5-15. https://doi.org/10.1111/j.1365-2958.2006.05072.x.

Vos, M., and G. J. Velicer. 2006. "Genetic population structure of the soil bacterium Myxococcus xanthus at the centimeter scale." Applied and Environmental Microbiology 72: 3615-25. https://doi.org/10.1128/aem.72.5.3615-3625.2006. 
Vos, Michiel, and Gregory J. Velicer. 2009. "Social conflict in centimeter- and global-scale populations of the bacterium Myxococcus xanthus." Current Biology 19: 1763-67. https://doi.org/10.1016/j.cub.2009.08.061.

Votaw, Heather R., and E. A. Ostrowski. 2017. "Stalk size and altruism investment within and among populations of the social amoeba." Journal of Evolutionary Biology 30: 2017-30. https://doi.org/10.1111/jeb.13172.

Yang, Z. and P. I. Higgs. 2014. "Myxobacteria : genomics, cellular and molecular biology.". Caister Academic Press.

Wei, Xueming, Darshankumar T. Pathak, and Daniel Wall. 2011. "Heterologous protein transfer within structured myxobacteria biofilms." Molecular Microbiology 81: 315-26. https://doi.org/10.1111/j.1365-2958.2011.07710.x.

West, Stuart A., Roberta M. Fisher, Andy Gardner, and E. Toby Kiers. 2015. "Major evolutionary transitions in individuality." Proceedings of the National Academy of Sciences U.S.A. 112: 10112-19. https://doi.org/10.1073/pnas.1421402112.

Wielgoss, Sébastien, Rebekka Wolfensberger, Lei Sun, Francesca Fiegna, and Gregory J. Velicer. 2019. "Social genes are selection hotspots in kin groups of a soil microbe." Science 363: 1342-1345. https://doi.org/10.1126/science.aar4416.

Wilson, H. V. 1907. "On some phenomena of coalescence and regeneration in sponges." Journal of Experimental Zoology 5: 245-58. https://doi.org/10.1002/jez.1400050204.

Wireman, J. W., and M. Dworkin. 1977. "Developmentally induced autolysis during fruiting body formation by Myxococcus xanthus." Journal of Bacteriology 129 (2): 798-802.

Zhang, Zheren, Dennis Claessen, and Daniel E. Rozen. 2016. "Understanding microbial divisions of labor." Frontiers in Microbiology 7: 2070. https://doi.org/10.3389/fmicb.2016.02070. 